WILDLIFE DISEASE

\section{Red squirrels in the British Isles are infected with leprosy bacilli}

\author{
Charlotte Avanzi, ${ }^{1 *}$ Jorge del-Pozo, ${ }^{2 *}$ Andrej Benjak, ${ }^{1 *}$ Karen Stevenson, ${ }^{3}$ \\ Victor R. Simpson, ${ }^{4}$ Philippe Busso, ${ }^{1}$ Joyce McLuckie, ${ }^{3}$ Chloé Loiseau, ${ }^{1} \dagger$ \\ Colin Lawton, ${ }^{5}$ Janne Schoening, ${ }^{6}$ Darren J. Shaw, ${ }^{2}$ Jérémie Piton, ${ }^{1}$ \\ Lucio Vera-Cabrera, ${ }^{7}$ Jesùs S. Velarde-Felix, ${ }^{7}$ Fergal McDermott, ${ }^{6}$ \\ Stephen V. Gordon, ${ }^{6,8,9,10}$ Stewart T. Cole, ${ }^{1} \ddagger$ Anna L. Meredith ${ }^{2} \ddagger$
}

Leprosy, caused by infection with Mycobacterium leprae or the recently discovered Mycobacterium lepromatosis, was once endemic in humans in the British Isles. Red squirrels in Great Britain (Sciurus vulgaris) have increasingly been observed with leprosy-like lesions on the head and limbs. Using genomics, histopathology, and serology, we found $M$. lepromatosis in squirrels from England, Ireland, and Scotland, and $M$. leprae in squirrels from Brownsea Island, England. Infection was detected in overtly diseased and seemingly healthy animals. Phylogenetic comparisons of British and Irish $M$. lepromatosis with two Mexican strains from humans show that they diverged from a common ancestor around 27,000 years ago, whereas the $M$. leprae strain is closest to one that circulated in Medieval England. Red squirrels are thus a reservoir for leprosy in the British Isles.

0 ften considered a disease of the past, leprosy remains a public health problem in certain low- and middle-income countries, with $\sim 220,000$ new cases reported annually (1). Leprosy was rife in Europe in the Middle Ages but disappeared during the 15th and 16th centuries, probably because of social segregation, other infectious diseases such as plague, or changes in host immunity (2-5). Today, all British clinical cases occur in individuals with a history of residence in a leprosy-endemic country (6). The disease manifests in different forms, ranging from multibacillary (or lepromatous) to paucibacillary (or tuberculoid), depending on the immunogenetics of the host (4). In all forms, skin lesions are accompanied by peripheral nerve damage, which causes sensory loss and may lead to deformities.

It had generally been accepted that leprosy resulted solely from interhuman transmission of Mycobacterium leprae. But in recent years, compelling evidence emerged from the south-

${ }^{1}$ Global Health Institute, Ecole Polytechnique Fédérale de Lausanne, 1015 Lausanne, Switzerland. ${ }^{2}$ Royal (Dick) School of Veterinary Studies and Roslin Institute, University of Edinburgh, Easter Bush Campus, Roslin, Scotland, UK. ${ }^{3}$ Moredun Research Institute, Pentlands Science Park, Bush Loan, Edinburgh, Scotland, UK. ${ }^{4}$ Wildlife Veterinary Investigation Centre, Chacewater, Cornwall, UK. ${ }^{5}$ School of Natural Sciences, Ryan Institute, National University of Ireland, Galway, Ireland. UCD School of Veterinary Medicine. University College Dublin, Belfield, Dublin, Ireland. 'Laboratorio Interdisciplinario de Investigación Dermatológica, Servicio de Dermatología, Hospital Universitario, Monterrey, N.L., Mexico. ${ }^{8}$ UCD School of Medicine, University College Dublin, Belfield, Dublin, Ireland. ${ }^{9}$ UCD School of Biomolecular and Biomedical Science, University College Dublin, Belfield, Dublin, Ireland ${ }^{10}$ UCD Conway Institute of Biomolecular and Biomedical Research, University College Dublin, Belfield, Dublin, Ireland. *These authors contributed equally to this work. †Present address: Department of Medical Parasitology and Infection Biology, Swiss Tropical and Public Health Institute, 4002 Basel, Switzerland. $\ddagger$ Corresponding author: Email: stewart.cole@epfl.ch (S.T.C.); anna.meredith@ed.ac.uk (A.L.M.) ern United States for zoonotic cases after exposure to infected nine-banded armadillos (Dasypus novemcinctus) (7-9). Furthermore, M. leprae was considered to be the sole causative agent of leprosy until 2008, when a new species, Mycobacterium lepromatosis, was identified in patients with diffuse lepromatous leprosy (DLL) (10). Such cases were primarily associated with Mexico and the Caribbean region (11). Comparison of the genome sequences of $M$. lepromatosis and $M$. leprae revealed that despite separating millions of years ago, the two genomes are remarkably similar in their size, organization, and (pseudo)gene content, but show only $88 \%$ sequence identity (11).

The Eurasian red squirrel Sciurus vulgaris is a widespread Palearctic species found from Ireland in the west to Kamchatka in the east $(12,13)$. However, in the United Kingdom, the $S$. vulgaris population of $\sim 140,000$ is severely threatened by habitat loss, squirrel poxvirus infection, and competition with $>2.5$ million gray squirrels, Sciurus carolinensis, introduced from North America $(14,15)$. Because of their endangered status, red squirrels are now protected (16). Recent detection of mycobacterial infection in red squirrels was reported in Scotland, with lesions and histopathology characteristic of DLL and evidence for $M$. lepromatosis being the etiological agent (17). Similarly affected squirrels were observed on the Isle of Wight and Brownsea Island in southern England (18), and observations of squirrel leprosy in Scotland are increasing (Fig. 1). Here, we investigated the leprosy outbreak using 70 red squirrel cadavers from Great Britain, with or without disease signs; 40 cadavers from Ireland, where no sightings of squirrels with leprosy signs have been reported; and four Scottish gray squirrel cadavers.

A differential polymerase chain reaction (PCR) screen was implemented to detect $M$. leprae and
M. lepromatosis DNA (11). We analyzed a total of 172 tissue samples from 13 animals with leprosy features and 101 without leprosy features (tables S1 and S2) (19). Six Scottish squirrels (two without clinical signs) (17), two from Ireland (no clinical signs), and one from the Isle of Wight, England (18) contained M. lepromatosis in several tissue samples from different anatomical sites, whereas all 25 red squirrels (17 without clinical signs) tested from Brownsea Island were infected with $M$. leprae (Fig. 1 and table S3). No cases of co-infection were observed (table S3). From the combined results, we concluded that $21 \%$ [21/101; 95\% confidence interval (CI), 13 to $30 \%]$ of the squirrels without clinical signs and all 13 of the animals with clinical signs harbored leprosy bacilli.

Serological tests were performed on nine diseased and 14 healthy red squirrels from Scotland and England, as well as the four gray squirrels. The grays were all seronegative, whereas 13 of 23 blood samples from red squirrels contained antibodies for the leprosy-specific antigen PGL-1 (phenolic glycolipid-1) (20) (table S4). Serology is useful to confirm the disease and to predict infection in live animals but cannot be used for species identification, as both $M$. leprae and $M$. lepromatosis produce this cell wall antigen (11).

Diseased Scottish squirrels infected with $M$. lepromatosis displayed a range of macroscopic lesions, including alopecia and extensive swelling of the snout, lips, eyelids, ear pinnae, and limb extremities (Fig. 1, Fig. 2A, fig. S1, and tables S2 and S5) (19). Histopathological examination of four such squirrels (Fig. 2B) revealed granulomatous dermatitis, sheets of epithelioid macrophages, and large numbers of acid-fast bacilli (AFB). There was neural involvement with the presence of AFB in nerve endings; neuritis was patchy and more frequently perineural (Fig. 2C). Inflammation was not focused exclusively around nerves and was mostly dermal. There were no signs of vasculitis, but AFB were present intravascularly (Fig. 2C). Similar lesions were observed in eight squirrels from Brownsea Island infected with $M$. leprae, although these animals also harbored numerous AFB in the spleen (Fig. 2C). Overall, the macroscopic signs and histopathology were characteristic of lepromatous leprosy (Fig. 2, A and B, and figs. S2 and S3). From post mortem inspection of diseased squirrels, it was not possible to distinguish between infection with $M$. lepromatosis or $M$. leprae, as is the case in human leprosy $(11,21,22)$.

To obtain deeper insight into the strains responsible and to perform phylogenetic analyses, we used a variety of DNA enrichment techniques (table S6) prior to Illumina sequencing, which was necessary because neither $M$. leprae nor $M$. lepromatosis can be cultured (19). Sufficient sequence coverage of $M$. lepromatosis genomes from seven squirrels was obtained (table S7). In parallel, we sequenced an additional genome of M. lepromatosis, Pl-02, from a PGL-1-seropositive patient from Sinaloa, Mexico (tables S1 and S4). The resultant sequence reads were mapped against the reference $M$. lepromatosis genome 
sequence from a patient from Monterrey, Mexico (11) to identify polymorphisms. Consistent with previous $M$. leprae genome comparisons $(9,11,23)$, there was an exceptionally high level of sequence conservation between $M$. lepromatosis strains (99.99\% identity) despite their different geographic origins. The two Mexican patient isolates differed by only seven single-nucleotide polymorphisms (SNPs), whereas the number of SNPs in the six British and Irish strains ranged from 1 to 17 on pairwise comparisons (table S8). Overall, there are roughly 400 SNPs that distinguish $M$. lepromatosis strains from Mexico and the British Isles (table S8). Clustering of Mexican and British M. lepromatosis strains into two distinct lineages was supported by maximumparsimony (fig. S4) and neighbor-joining (fig. S5) phylogenetic reconstructions. On the basis of the $M$. leprae mutation rate (19) and using the Bayesian inference software BEAST (24), we estimated that the British Isles and Mexican strains diverged from their most recent common ancestor around 27,000 years ago, whereas the Irish and British strains diverged as recently as 200 years ago (Fig. 3A). The latter estimate is consistent with the date of the first campaign to reintroduce the red squirrel into Ireland from England between 1820 and 1856, following its extinction in the 17 th century $(12,25)$. This suggests that these animals may already have been infected with $M$. lepromatosis when they were reintroduced.

Finding $M$. leprae in red squirrels in England was unexpected, because leprosy was eradicated from the British Isles several centuries ago, thus demonstrating that a pathogen can persist in the environment long after its clearance from the human reservoir. Furthermore, this is only the second report of $M$. leprae in nonprimate species. From Bayesian and maximum-parsimony analysis (Fig. 3B and fig. S4A), we note that the two closest relatives to the strain of $M$. leprae found on Brownsea Island were both from medieval Europe. Intriguingly, one of these (SK2) originated from the skeletal remains of a leprosy victim buried about 730 years ago in Winchester, a city situated a mere $70 \mathrm{~km}$ from Brownsea Island (Fig. 1). Like SK2, the Brownsea Island strain of $M$. leprae belongs to sequence type 3I, which forms a distinct $M$. leprae branch (Fig. 3B) (3) and is now endemic in wild armadillos in the southern United States (9). Thus, M. leprae with this particular sequence type is capable of infecting at least three different hosts: humans, red squirrels, and armadillos.

Because there were no obvious genomic polymorphisms restricted to the $M$. leprae 3I type that might account for this broad host range (tables S9 and S10), we explored the possibility that these three species might share a major susceptibility gene and focused on TLR1. This candidate gene, encoding the surface-exposed Toll-like receptor 1 (TLR1) displayed on various epithelial and immune cells, is known to be associated with susceptibility to leprosy (Fig. 4A). A dysfunctional TLRI allele encoding an Ile ${ }^{602} \rightarrow$ Ser variant with an altered transmembrane domain is prevalent in Caucasians and is asso- ciated with a decreased risk for leprosy $(5,26)$. By contrast, the TLR1 $\mathrm{Asn}^{248} \rightarrow$ Ser variant is humans. This mutation is located in the ninth (LRR) region of TLR1 (27). Furthermore, in ninebanded armadillos, an $\mathrm{Arg}^{627} \rightarrow$ Gly change in TLR1 [close to the Toll-interleukin receptor (TIR) domain; Fig. 4A] seemingly confers resistance to leprosy (28). Using PCR, the coding exon of TLRI was amplified and sequenced from 58 red squirrels (with or without lesions) and three gray squirrels (tables S11, S12, and S14) (19). Upon comparison of the sequences and TLR1 associated with an increased risk of leprosy in repeat of the extracellular leucine-rich repeat

alignments (table S13), no polymorphisms were observed at the same sites associated with leprosy in humans and armadillos. However, in some red squirrels, two distinct polymorphic sites exist: a single SNP leading to a $\operatorname{Ser}^{494} \rightarrow$ Asn mutation in the 19th repeat of the LRR region, and a cluster of linked mutations that produce $\mathrm{Ser}^{657} \rightarrow$ Asn $(\mathrm{S} 657 \mathrm{~N})$, Leu $^{660} \rightarrow$ Val $(\mathrm{L} 660 \mathrm{~V})$, and Asn $^{662} \rightarrow$ Cys (N662C) variants in helix 1 of the TIR domain (Fig. 4B). These mutations were found less frequently in squirrels infected with leprosy bacilli than in healthy animals, which suggests that they may confer protection (for $\mathrm{S} 494 \mathrm{~N}$, odds ratio $=5.77 ; 95 \%$ CI, 1.42 to 23.41 ;

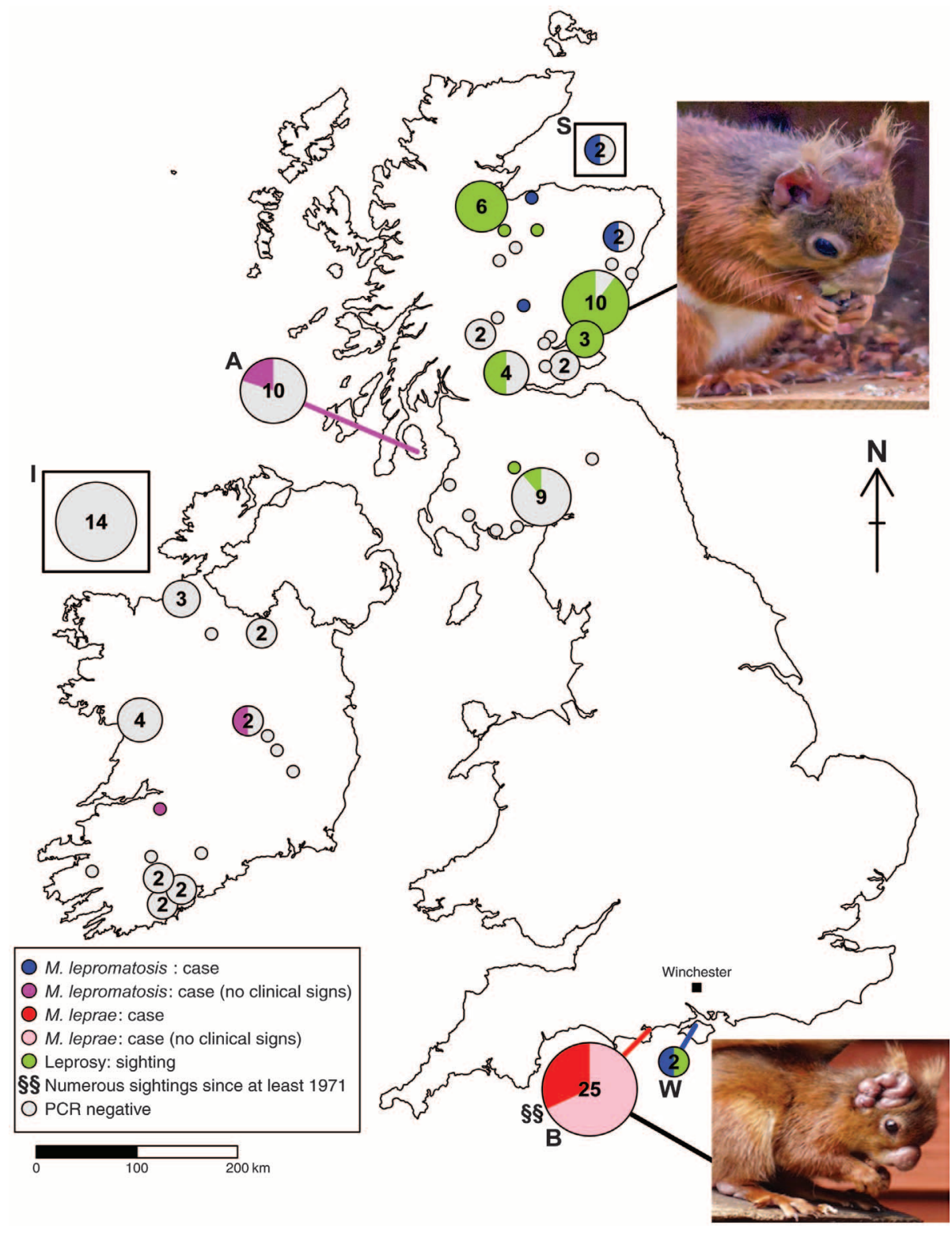

Fig. 1. Squirrel sampling sites in the British Isles. Pie charts indicate the location of sites where squirrels were sighted or found and are color-coded as indicated in the box; numbers within circles indicate different animals tested where $N>1$. Boxed circles refer to squirrels of unknown location. I, Ireland; S, Scotland; A, Isle of Arran; B, Brownsea Island; W, Isle of Wight. The figure was drawn in R (v3.2.23) with the package maps (v3.1.0) using the mapdata (v2.2-6) "worldHiresMapEnv" and the package plotrix (v3.6-2) for pie charts. 
A

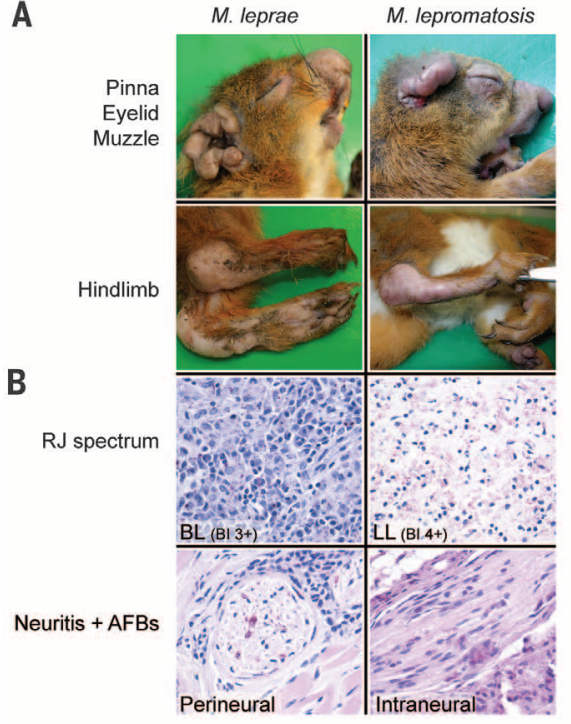

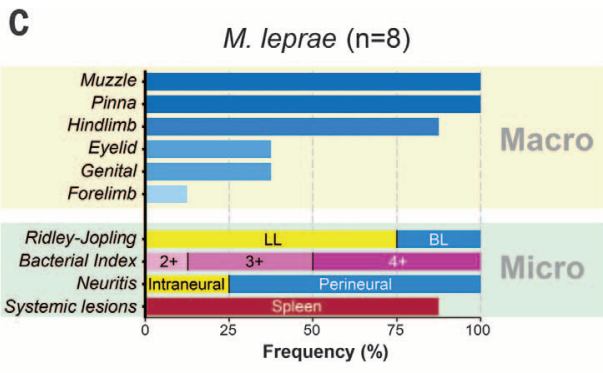

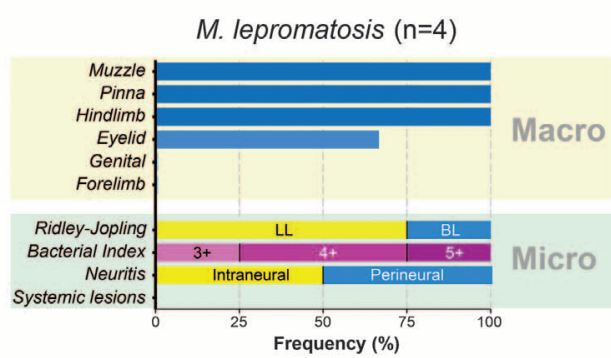

Fig. 2. Gross histopathological features of red squirrels with leprosy. (A) Macroscopic features of squirrels infected with M. lepromatosis or M. leprae are similar. (B) Histological examination of tissue sections from infected squirrels using the Ridley-Jopling (RJ) classification after Ziehl Neelsen staining (magnification 400x). LL, lepromatous leprosy; BL, borderline lepromatous leprosy. (C) Summary of main macroscopic and microscopic findings from squirrels infected with M. leprae $(n=8)$ or M. lepromatosis $(n=4)$.

A

M. lepromatosis

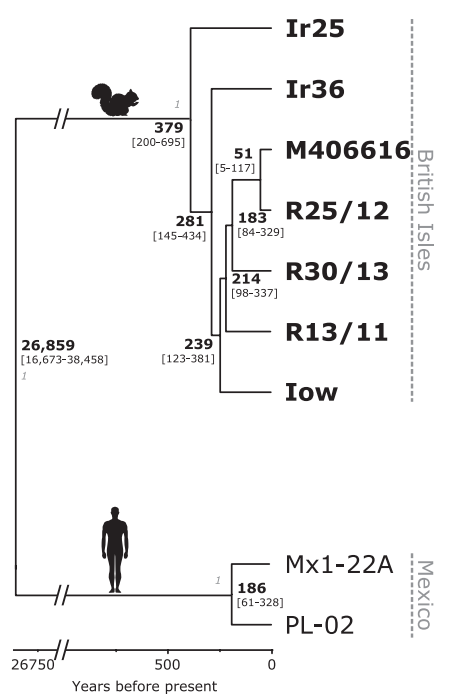

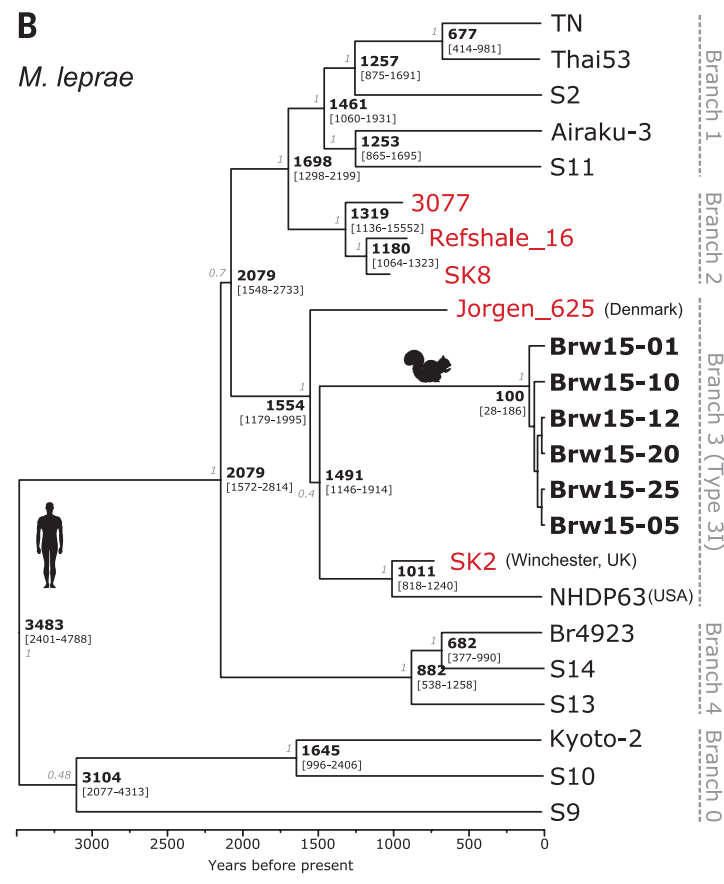

Fig. 3. Phylogeny of leprosy bacilli. (A) Bayesian phylogenetic tree representation of nine M. lepromatosis genome sequences obtained from squirrels (bold) or humans (upper and lower parts, respectively), calculated by BEAST 1.8.2 (24) using the mutation rate of $M$. leprae and inferred from 432 genome-wide variable positions. Squirrel sample prefixes: Ir, Ireland; low, Isle of Wight; all others from Scotland. Both human strains were from Mexico. (B) Bayesian phylogenetic tree representation of $M$. leprae inferred from 498 genome-wide variable positions, calculated as in (A). For squirrel samples (bold), Brw denotes Brownsea Island cluster; red labeling indicates ancient strains for which radiocarbon dating information was available (3). For both trees, divergence time intervals are shown on each node in years before present, with the $95 \%$ highest posterior density (HPD) range in brackets. Posterior probabilities for each node are shown in gray.

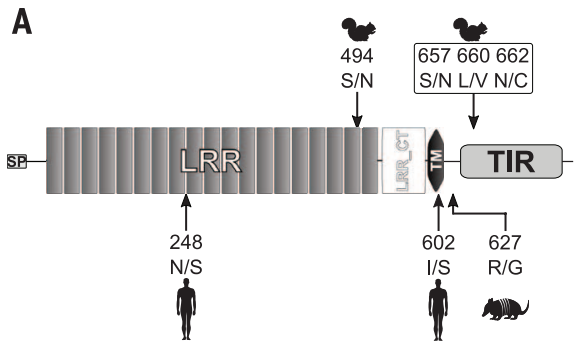

B

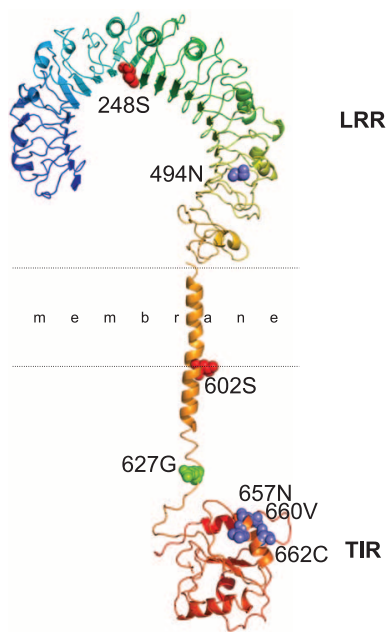

Fig. 4. Organization, structure, and polymorphisms in TLR1 associated with leprosy in humans, armadillos, and red squirrels. (A) Schematic representation of TLR1 and its domains (drawn to scale). SP, signal peptide; LRR, leucinerich repeat; LRR_CT, leucine-rich repeat C-terminal; $\mathrm{TM}$, transmembrane domain; TIR, Toll/interleukin1 receptor. (B) Structural model of the red squirrel TLR1. Protein is colored in a rainbow spectrum from $\mathrm{N}$ terminus (blue) to $\mathrm{C}$ terminus (red). Amino acid abbreviations: C, Cys; G, Gly; I, Ile; L, Leu; N, Asn; R, Arg; S, Ser; V, Val.

$P=0.01$; for S657N-L660V-N662C, odds ratio = 4.89 ; $95 \%$ CI, 0.98 to $24.53 ; P=0.05$ ).

It is unclear whether leprosy is contributing to the demise of the red squirrel population or how these animals became infected with $M$. lepromatosis or $M$. leprae. Because $M$. lepromatosis has only recently been discovered as a human pathogen (10) and few detailed case reports have been published $(10,11,21,29)$, further investigation is required to establish its relative prevalence in wildlife compared to humans. M. leprae was long considered to be an obligate human pathogen that was introduced to the Americas by European settlers, prior to anthroponotic infection of armadillos, because there are no human skeletal remains with signs of leprosy from the pre-Columbian era (9). The discovery that the strain of M. leprae in red squirrels on Brownsea Island today is essentially the same as one that circulated in medieval England and Denmark, and is highly related to the extant North American armadillo strain, raises the possibility of a second anthroponotic introduction in Europe. If this were 
the case, it must have occurred several centuries ago, as leprosy became increasingly scarce in the British Isles after the 17th century (3). It is also conceivable that humans may have been infected through contact with red squirrels bearing $M$. leprae, as these animals were prized for their fur and meat in former times (30). Our findings show that further surveys of animal reservoirs of leprosy bacilli are warranted, because zoonotic infection from such reservoirs may contribute to the inexplicably stubborn plateau in the incidence of the human leprosy epidemic despite effective and widespread treatment with multidrug therapy (1).

\section{REFERENCES AND NOTES}

1. World Health Organization, Wkly. Epidemiol. Rec. 88, 365-379 (2013).

2. H. D. Donoghue et al., Infect. Genet. Evol. 31, 250-256 (2015).

3. V. J. Schuenemann et al., Science 341, 179-183 (2013).

4. A. Alter, A. Grant, L. Abel, A. Alcaïs, E. Schurr, Mamm. Genome 22, 19-31 (2011)

5. S. H. Wong et al., PLOS Pathog. 6, e1000979 (2010).

6. N. Fulton, L. F. Anderson, J. M. Watson, I. Abubakar, BMJ Open 6. $\mathrm{e} 010608$ (2016).

7. R. Sharma et al., Emerg. Infect. Dis. 21, 2127-2134 (2015).

8. R. Truman, Lepr. Rev. 76, 198-208 (2005)

9. R. W. Truman et al., N. Engl. J. Med. 364, 1626-1633 (2011).

10. X. Y. Han et al., Am. J. Clin. Pathol. 130, 856-864 (2008).

11. P. Singh et al., Proc. Natl. Acad. Sci. U.S.A. 112, 4459-4464 (2015).

12. M. Carey, G. Hamilton, A. Poole, C. Lawton, The Irish Squirrel Survey 2007 (COFORD, Dublin, 2007)

13. S. Harris, G. B. Corbet, The Handbook of British Mammals (Mammal Society/Blackwell Scientific, ed. 3, 1991).

14. D. M. Tompkins, A. W. Sainsbury, P. Nettleton, D. Buxton, J. Gurnell, Proc. R. Soc. B 269, 529-533 (2002).

15. E. Stokstad, Science 352, 1268-1271 (2016).

16. Council of Europe, Convention on the Conservation of European Wildife and Natural Habitats (ETS No. 104), Appendix III (1979). https://rm.coe.int/CoERMPublicCommonSearchServices/ DisplayDCTMContent?documentld=0900001680304356

17. A. Meredith et al., Vet. Rec. 175, 285-286 (2014).

18. V. Simpson et al., Vet. Rec. 177, 206-207 (2015).

19. See supplementary materials on Science Online.

20. J. S. Spencer, P. J. Brennan, Lepr. Rev. 82, 344-357 (2011).

21. J. S. Velarde-Félix, G. Alvarado-Villa, L. Vera-Cabrera, Am. J. Trop. Med. Hyg. 94, 483-484 (2016).

22. L. Vera-Cabrera et al., J. Clin. Microbiol. 53, 1945-1946 (2015).

23. M. Monot et al., Nat. Genet. 41, 1282-1289 (2009).

24. A. J. Drummond, A. Rambaut, BMC Evol. Biol. 7, 214 (2007).

25. B. P. Vieira, C. Fonseca, R. G. Rocha, Anim. Biodivers. Conserv. 38, 49-58 (2015)

26. S. R. Krutzik et al., Nat. Med. 9, 525-532 (2003).

27. C. de Sales Marques et al., J. Infect. Dis. 208, 120-129 (2013).

28. L. B. Adams et al., Mem. Inst. Oswaldo Cruz 107 (suppl. 1), 197-208 (2012).

29. P. G. Jessamine et al., J. Drugs Dermatol. 11, 229-233 (2012).

30. P. Lurz, Red Squirrel: Naturally Scottish (Scottish Natural Heritage, 2010)

\section{ACKNOWLEDGMENTS}

We thank E. Sheehy, E. Goldstein, M. Flaherty, A. Zintl, the National Trust, Forestry Commission Scotland, and Saving Scotland's Red Squirrels for samples, help, and advice. We thank the Genomic Technologies Facility at the University of Lausanne for Illumina sequencing and technical support. Raw sequence read files have been deposited in the Sequence Read Archive of the National Center for Biotechnology Information under accession numbers SRR3672737 to SRR3672758 (NCBI BioProject PRJNA325727), SRR3674396 to SRR3674450 (NCBI BioProject PRJNA325827), SRR3674451 to SRR3674453 (NCBI BioProject PRJNA325856), and SRR3673933; representative TLR1 sequences have been deposited in GenBank under accession numbers KX388139, KX388140, and KX388141. Phylogenetic trees and SNP alignments have been deposited at Treebase under Study Accession URL http://purl.org/phylo/ treebase/phylows/study/TB2:S19692. Supported by the Fondation Raoul Follereau and Swiss National Science Foundation grant IZRJZ3_164174 (S.T.C.), the Scottish Government Rural and
Environment Science and Analytical Services Division (K.S.), and the Thomas O'Hanlon Memorial Award in Veterinary Medicine (F.McD.).

\section{SUPPLEMENTARY MATERIALS}

www.sciencemag.org/content/354/6313/744/suppl/DC1 Materials and Methods

Figs. S1 to S5

Tables S1 to S14

References (31-51)

21 June 2016; accepted 27 September 2016

10.1126/science.aah3783

\title{
ARCTIC SEA ICE
}

\section{Observed Arctic sea-ice loss directly follows anthropogenic $\mathrm{CO}_{2}$ emission}

\author{
Dirk Notz ${ }^{1 *}$ and Julienne Stroeve ${ }^{2,3}$
}

Arctic sea ice is retreating rapidly, raising prospects of a future ice-free Arctic Ocean during summer. Because climate-model simulations of the sea-ice loss differ substantially, we used a robust linear relationship between monthly-mean September sea-ice area and cumulative carbon dioxide $\left(\mathrm{CO}_{2}\right)$ emissions to infer the future evolution of Arctic summer sea ice directly from the observational record. The observed linear relationship implies a sustained loss of $3 \pm 0.3$ square meters of September sea-ice area per metric ton of $\mathrm{CO}_{2}$ emission. On the basis of this sensitivity, Arctic sea ice will be lost throughout September for an additional 1000 gigatons of $\mathrm{CO}_{2}$ emissions. Most models show a lower sensitivity, which is possibly linked to an underestimation of the modeled increase in incoming longwave radiation and of the modeled transient climate response.

$\mathrm{T}$ he ongoing rapid loss of Arctic sea ice has far-reaching consequences for climate, ecology, and human activities alike. These include amplified warming of the Arctic (1), possible linkages of sea-ice loss to midlatitude weather patterns (2), changing habitat for flora and fauna (3), and changing prospects for human activities in the high north (3). To understand and manage these consequences and their possible future manifestation, we need to understand the sensitivity of Arctic sea-ice evolution to changes in the prevailing climate conditions. However, assessing this sensitivity has been challenging. For example, climate-model simulations differ widely in their timing of the loss of Arctic sea ice for a given trajectory of anthropogenic $\mathrm{CO}_{2}$ emissions: Although in the most recent Climate Model Intercomparison Project 5 (CMIP5) (4), some models project a near icefree Arctic during the summer minimum already toward the beginning of this century, other models keep a substantial amount of ice well into the next century even for an external forcing based on largely undamped anthropogenic $\mathrm{CO}_{2}$ emissions as described by the Representative Concentration Pathway scenario RCP8.5 $(4,5)$.

To robustly estimate the sensitivity of Arctic sea ice to changes in the external forcing, we

${ }^{1}$ Max Planck Institute for Meteorology, Hamburg, Germany.

${ }^{2}$ National Snow and Ice Data Center, Boulder, CO, USA.

${ }^{3}$ University College, London, UK.

*Corresponding author. Email: dirk.notz@mpimet.mpg.de identify and examine a fundamental relationship in which the CMIP5 models agree with the observational record: During the transition to a seasonally ice-free Arctic Ocean, the 30-year running mean of monthly mean September Arctic sea-ice area is almost linearly related to cumulative anthropogenic $\mathrm{CO}_{2}$ emissions (Fig. 1). In the model simulations, the linear relationship holds until the 30-year running mean, which we analyze to reduce internal variability, samples more and more years of a seasonally ice-free Arctic Ocean, at which point the relationship levels off toward zero. For the first few decades of the simulations, a few models simulate a nearconstant sea-ice cover despite slightly rising cumulative $\mathrm{CO}_{2}$ emissions. This suggests that in these all-forcing simulations, greenhouse-gas emissions were initially not the dominant driver of sea-ice evolution. This notion is confirmed by the CMIP5 $1 \% \mathrm{CO}_{2}$ simulations, where the initial near-constant sea-ice cover does not occur (fig. S3A). With rising greenhouse-gas emissions, the impact of $\mathrm{CO}_{2}$ becomes dominating also in all all-forcing simulations, as evidenced by the robust linear trend that holds in all simulations throughout the transition period to seasonally icefree conditions. We define this transition period as starting when the 30-year mean September Arctic sea-ice area in a particular simulation decreases for the first time to an area that is $10 \%$ or more below the simulation's minimum sea-ice cover during the period 1850 to 1900 , and 


\section{Science IAAAS}

Red squirrels in the British Isles are infected with leprosy bacilli Charlotte Avanzi, Jorge del-Pozo, Andrej Benjak, Karen Stevenson, Victor R. Simpson, Philippe Busso, Joyce McLuckie, Chloé Loiseau, Colin Lawton, Janne Schoening, Darren J. Shaw, Jérémie Piton, Lucio Vera-Cabrera, Jesùs S. Velarde-Felix, Fergal McDermott, Stephen V. Gordon, Stewart T. Cole and Anna L. Meredith (November 10, 2016) Science 354 (6313), 744-747. [doi: 10.1126/science.aah3783]

Editor's Summary

\section{British squirrels infected with leprosy}

With the exception of armadillos in the Americas, leprosy infections are considered almost exclusively restricted to humans. Avanzi et al. examined warty growths on the faces and extremities of red squirrels in the British Isles and found that two species of leprosy-causing organisms were to blame (see the Perspective by Stinear and Brosch). Mycobacterium leprae in the southern population of Brownsea Island squirrels originated from a medieval human strain. M. lepromatosis was found in red squirrels from elsewhere in the United Kingdom and Ireland. Human leprosy is proving hard to eradicate, despite available drugs. Perhaps other wildlife species are also reservoirs for this stubborn disease.

Science, this issue p. 744 ; see also p. 702

This copy is for your personal, non-commercial use only.

Article Tools Visit the online version of this article to access the personalization and article tools:

http://science.sciencemag.org/content/354/6313/744

Permissions Obtain information about reproducing this article: http://www.sciencemag.org/about/permissions.dtl

Science (print ISSN 0036-8075; online ISSN 1095-9203) is published weekly, except the last week in December, by the American Association for the Advancement of Science, 1200 New York Avenue NW, Washington, DC 20005. Copyright 2016 by the American Association for the Advancement of Science; all rights reserved. The title Science is a registered trademark of AAAS. 